\title{
ANÁLISE DA ASSOCIAÇÃO ENTRE MAPAS DO MONITOR DE SECAS E DADOS CLIMÁTICOS DE SERGIPE
}

\author{
SANTOS, Kelly Marina Silva - kmsskelly@gmail.com \\ Universidade Federal de Sergipe / UFS
}

\author{
ALBUQUERQUE, Tatiana Máximo Almeida - tatianamaximoalmeida@gmail.com \\ Universidade Federal de Sergipe / UFS
}

MENDES, Ludmilson Abritta - lamendes@ufs.br

Universidade Federal de Sergipe / UFS

Submetido em: 11/05/2020

Aceito para publicação em: 17/05/2021

Publicado em: 01/06/2021

DOI: http://dx.doi.org/10.5380/abclima.v28i0.73648

\begin{abstract}
RESUMO: A seca é um fenômeno natural recorrente em diversas regiões do planeta e provoca sérios impactos sobre o meio ambiente e a população. O histórico da gestão de secas no Brasil revela o caráter emergencial e reativo das medidas comumente adotadas. Contudo, a implementação do Monitor de Secas (MSB) no país evidencia o início da transição para gestão de riscos. Essa ferramenta consiste em um produto de monitoramento baseado em diversos índices e dados observados, através de um processo participativo entre instituições federais e estaduais. Dada a importância da consolidação do Monitor como meio de suporte à decisão para políticas públicas, esta pesquisa buscou avaliar a existência de associação entre as categorias de seca do MSB e dados climáticos disponíveis para a validação no estado de Sergipe. Foram utilizadas as ferramentas estatísticas do teste de independência do Qui-Quadrado e do coeficiente de correlação $V$ de Cramer. $O$ teste de independência mostrou que há associação entre os mapas do MSB e as precipitações mensais, trimestrais e semestrais; anomalias mensais, trimestrais e semestrais; e o Índice de Vegetação da Diferença Normalizada (NDVI). Contudo, o cálculo do coeficiente de Cramer identificou fraqueza na associação com os dados de precipitação e anomalias, com melhores resultados à medida que o período considerado foi maior. O NDVI foi a variável climática com maior correlação com as categorias do Monitor, refletindo características agrícolas da seca. De modo geral, podese afirmar que os dados disponíveis para a validação em Sergipe têm baixa associação com os mapas do Monitor, sendo necessária a inserção de novos materiais de apoio, semelhante aos produtos de apoio utilizados na elaboração dos mapas do MSB.
\end{abstract}

PAlaVRAS-ChaVe: Monitoramento. Gestão de Secas. Políticas Públicas.

\section{EVALUATION OF ASSOCIATION BETWEEN DROUGHT MONITOR MAPS AND SERGIPE CLIMATE DATA}

ABSTRACT: Drought is a recurring natural phenomenon in several regions around the world and causes serious effects on the environment and the population. The history of drought management in Brazil reveals the emergency and reactive nature of the adopted measures. However, the implementation of Drought Monitor (MSB) shows the beginning of a transition to risk management in the country. This tool consists of a drought monitoring based on multiple indices and observed data, through a participatory process between federal and state institutions. Due to the importance of consolidating the Monitor as a means to support the decision for public policies, this research aimed to evaluate the existence of association between MSB drought categories and climatic data for the state of Sergipe. The statistical tools used for the analysis were the Chi-Square independence test and the Cramer's $V$ coefficient. The independence test showed that there is association between MSB maps and monthly, quarterly and half-yearly rainfall; monthly, quarterly and half-yearly anomalies; and the Normalized Difference Vegetation Index (NDVI). However, the results of Cramer's coefficient identified weak association 
with precipitation and anomalies and better results as the considered period was longer. NDVI was the climatic variable with the highest correlation with Monitor categories, reflecting agricultural drought characteristics. In general, this work concluded that the available data for validation in Sergipe have a low association with the Monitor maps, requiring the insertion of new information, as the support products used in the elaboration of the MSB maps.

KEYWORDS: Monitoring. Drought Management. Public Policy

\section{EVALUACIÓN DE LA ASOCIACIÓN ENTRE MAPAS DEL MONITOR DE LA SEQUÍA Y DATOS CLIMÁTICOS DE SERGIPE}

RESUMEN: La sequía es un fenómeno natural recurrente en varias regiones del planeta y causa graves impactos en el medio ambiente y la población. La historia del manejo de la sequía en Brasil revela la emergencia y la naturaleza reactiva de las medidas adoptadas. Sin embargo, la implementación del Monitor de sequía (MSB) en el país muestra el comienzo de la transición a la gestión de riesgos. Esta herramienta consiste en un producto de monitoreo basado en varios índices y datos observados, a través de un proceso participativo entre instituciones federales y estatales. Dada la importancia de consolidar el Monitor como un medio de apoyar la toma de decisiones para las políticas públicas, esta investigación buscó evaluar la existencia de una asociación entre las categorías de sequía en el MSB y los datos climáticos para el estado de Sergipe. Se utilizaron las herramientas estadísticas de la prueba de independencia Chi-Square y el coeficiente de correlación $\mathrm{V}$ de Cramer. La prueba de independencia mostró que existe una asociación entre los mapas MSB y las precipitaciones mensuales, trimestrales y semestrales; anomalías mensuales, trimestrales y semestrales; y el Índice de Vegetación de Diferencia Normalizada (NDVI). Sin embargo, el cálculo del coeficiente de Cramer identificó debilidad en la asociación con datos sobre precipitación y anomalías, con mejores resultados ya que el período considerado fue más largo. NDVI fue la variable climática con la mayor correlación con las categorías de Monitor, reflejando las características de la sequía agrícola. En general, se puede decir que los datos disponibles para validación en Sergipe tienen poca asociación con los mapas de Monitor, requiriendo la inserción de nuevos materiales de soporte, similares a los productos de soporte utilizados en la elaboración de los mapas del MSB.

PALABRAS ClAVE: Monitoreo. Manejo de sequías. Políticas públicas..

\section{INTRODUÇÃO}

A seca é um fenômeno natural complexo, podendo ser originada por múltiplos mecanismos ou fatores contribuintes, a exemplo de déficits de precipitação e aumentos de temperatura e evapotranspiração (HAO et al., 2018; KIEM et al., 2016). Uma das maiores dificuldades no estudo da seca decorre da falta de uma definição específica e mundialmente reconhecida. Visto que existem inúmeras definições, a existência da seca e seu grau de severidade devem ser determinados por região, considerando-se características climáticas e os impactos resultantes do período seco, estes relacionados às vulnerabilidades locais, à capacidade de resposta e às políticas de mitigação (CUNHA et al., 2019; WMO, 2006).

Historicamente, regiões com episódios de seca recorrentes costumam combatê-la através de medidas emergenciais, buscando alívio para a população por meio do fortalecimento da infraestrutura hídrica e do fornecimento de água, alimento e ração para o gado, como destaca o trabalho de Soares e Barbosa (2019). Contudo, esse tipo de ações, característico de gestão de crise, apenas ameniza os efeitos já consolidados da seca, não contribuindo para o aumento de resiliência da população visando a ocorrências futuras. 
Considerando-se a necessidade de evoluir para a gestão de riscos, monitorando eventos extremos e dando suporte às políticas públicas no país, faz-se necessário conhecer a variabilidade espaço-temporal de dados climatológicos (SANTOS et al., 2019). Nesse contexto, o Brasil, marcado por um longo histórico de medidas emergenciais, tem somado esforços estaduais e federais para melhorar a política de secas no país. Em 2014, entrou em operação no país o Monitor de Secas (MSB), ferramenta de monitoramento baseada no Monitor de Secas dos Estados Unidos (USDM, United States Drought Monitor), envolvendo a Agência Nacional de Águas (ANA) e outras instituições federais e estaduais, com objetivo de representar a intensidade atual da seca no Nordeste (inicialmente) e promover um referencial comum entre a União e os estados para a aplicação de políticas públicas (ANA, 2019).

O MSB consiste na representação das condições de seca da região Nordeste e outros estados através de um mapa elaborado mensalmente em um processo colaborativo. O processo de atualização mensal do MSB divide-se em quatro etapas: preparação dos dados, traçado do mapa, validação do Monitor e publicação do mapa.

Visando à melhor compreensão da severidade, permanência ou alívio de um período seco, inicialmente são reunidos índices de seca e produtos de apoio. Estes são dados obtidos de diversas instituições envolvidas, a exemplo da precipitação e da umidade do solo, enquanto aqueles são representações numéricas da seca por meio de cálculos estatísticos envolvendo dados climatológicos e hidrológicos regionais. Após o tratamento desses dados, os autores definem o novo traçado do mapa, baseado no mapa do mês anterior, nos índices de seca e nos produtos de apoio (BANCO MUNDIAL, 2015).

Um dos maiores diferenciais do Monitor em relação a outros produtos de monitoramento é a chamada fase de validação, em que o mapa é enviado aos estados participantes para confirmar ou refutar, com base em dados e informações locais, as definições de seca apresentadas. Assim, o processo de criação do Monitor torna-se participativo. Após as discussões entre autores e validadores, chegando a uma representação consensual da seca, os mapas são disponibilizados no site (MARTINS et al., 2016).

Em Sergipe, durante a etapa de validação, são utilizados dados de precipitação, anomalias de precipitação e do Índice de Vegetação da Diferença Normalizada (NDVI, Normalized Difference Vegetation Index) para analisar os aspectos meteorológico e agrícola da seca. Adicionalmente, informações socioeconômicas são analisadas por meio de um formulário, denominado Formulário Mínimo Padrão, respondido por observadores locais sobre as condições de seca vivenciadas pela população. Dessa forma, o mapa do Monitor de Secas proposto é aceito ou são sugeridas alterações baseadas em argumentos sólidos.

Outra peculiaridade do MSB é possuir como premissa a representação da seca por meio de uma visão macro, ou seja, é realizada uma análise por regiões climáticas e não por município (BANCO MUNDIAL, 2015), o que ocorre devido à insuficiência da distribuição de estações pluviométricas com dados precisos para uma representação micro em determinadas localidades. Como consequência, encontram-se dificuldades na etapa de verificação em Sergipe, menor estado do Nordeste, podendo resultar em discordância entre a informação disposta no mapa e a visão da instituição validadora estadual. Além disso, os índices de seca 
utilizados como fonte de dados para Sergipe baseiam-se em apenas três estações de monitoramento no estado, nenhuma delas situadas no Semiárido.

No estado de Sergipe, entre os anos 2016 e 2017, 29 municípios (38,7\% do total) decretaram estado de emergência em decorrência de seca ou estiagem, com impactos, sobretudo, na produção agrícola e no abastecimento de água para a população (ROCHA, 2017). Em 2018, foram 14 decretações. Em 2019, 25 municípios declararam situação de emergência por seca e um por estiagem, dentre eles os 7 municípios do Alto Sertão (SERGIPE, 2020).

Diante da importância do Monitor como instrumento de suporte à aplicação de políticas públicas, esta pesquisa teve como objetivo analisar a existência de associação entre as definições de seca apresentadas nos mapas do MSB e dados climáticos locais disponíveis para validação no estado de Sergipe, por meio da comparação entre as categorias de severidade indicadas nos mapas e os valores de precipitação e do NDVI.

\section{2. ÁREA DE ESTUDO}

Esta pesquisa tem como área de estudo o estado de Sergipe, que está situado na região Nordeste do Brasil, entre os paralelos 9031'S e 11033'S e os meridianos $36^{\circ} 25^{\prime} \mathrm{W}$ e $38^{\circ} 14^{\prime} \mathrm{W}$. Conhecido por ser o menor estado da Federação Brasileira, possui extensão territorial de $21.918,44 \mathrm{~km}^{2}$ e população estimada de 2.288.116 habitantes (SEPLAG, 2018).

O clima sergipano é caracterizado por temperaturas médias elevadas e pouca variação térmica anual, com amplitude média de $5^{\circ} \mathrm{C}$ em todo o estado e índices pluviométricos que diminuem do litoral para o interior. Baseado na temperatura e no regime pluvial, o estado pode ser dividido em três zonas climáticas distintas: Tropical Úmido ao Longo do Litoral (Leste), Tropical SubÚmido ou de Transição Semiárida (Agreste) e Semiárido (Sertão), representados na Figura 1 (SEPLAG, 2014?).
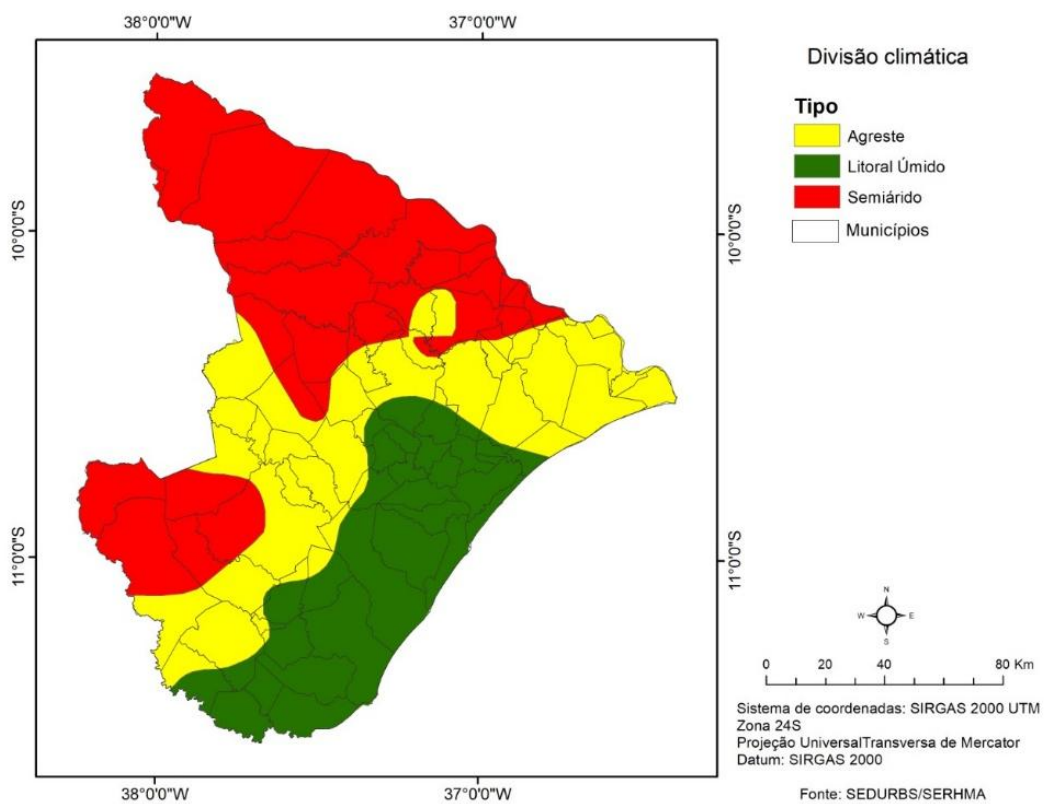

Figura 1 - Mapa de divisão climática de Sergipe. Fonte: DEPEC (2021). 
Dos 75 municípios sergipanos, 29 (equivalentes a 50,6\% do território estadual) encontram-se na região do Semiárido (SEPLAG, 2018; MI, 2017), em que há maior recorrência e severidade de secas no estado.

\section{MATERIAL E MÉTODOS}

Esta pesquisa foi realizada em duas etapas, descritas a seguir: a) aquisição e tratamento dos dados e b) aplicação da estatística e análise dos resultados.

\subsection{OBTENÇÃO E PREPARAÇÃO DOS DADOS} município:

Para a realização desta pesquisa, foram coletados os seguintes dados por

- Categorias mensais do Monitor de Secas;

- Acumulados mensais, trimestrais e semestrais de precipitação;

- Valores mensais, trimestrais e semestrais de anomalia de precipitação;

- Valores médios mensais de NDVI.

Todos os itens acima citados correspondem ao período de julho de 2018, quando teve início a validação em Sergipe, a outubro de 2019. Dados de precipitação, suas anomalias e NDVI foram utilizados neste trabalho por serem as principais fontes de informação de que os validadores dispõem durante a etapa de validação. Ressalta-se que, durante a validação, o ideal seria utilizar dados de suporte semelhantes àqueles utilizados durante a elaboração dos mapas, a exemplo dos índices de seca (para 1, 3, 6, 9, 12, 24 e 36 meses). Entretanto, à época da pesquisa, as informações utilizadas para validação correspondiam aos itens acima destacados.

Após a publicação dos mapas mensais, o site do Monitor de Secas (ANA, 2019) os disponibiliza para a sociedade em formato compatível com o programa de geoprocessamento QGIS. Por meio desse software, foi possível fazer um recorte de Sergipe a partir dos mapas originais. Além disso, foi adicionada uma camada correspondente à divisão do estado em municípios, como pode ser visto na Figura 2. Desse modo, foram identificados os níveis de severidade da seca (sem seca, fraca, moderada, grave, extrema, excepcional) para cada município e mês. Nos casos em que houve mais de uma categoria por município, foi considerada a condição mais rigorosa da seca, uma vez que o MSB deve orientar as políticas públicas e as populações mais vulneráveis aos impactos da seca devem ser prioritárias.

A Superintendência Especial de Recursos Hídricos e Meio Ambiente (SERHMA) forneceu, para esta pesquisa, os registros mensais de precipitação para os 25 postos pluviométricos ativos em Sergipe, bem como as séries históricas de cada um. A partir das séries históricas de precipitação mensal, foi possível gerar as séries históricas de anomalia, calculando-se a diferença entre o registro mensal e a média de longo prazo correspondente. De modo semelhante, foram geradas séries de precipitação trimestral e semestral (acumulando-se a precipitação dos três e seis últimos meses) e de anomalia de precipitação trimestral e semestral (diferença entre a média histórica do 
acumulado trimestral/semestral e o valor acumulado no último trimestre/semestre).

As falhas das séries históricas foram desconsideradas, uma vez que para a metodologia de cálculo adotada (descrita no Item 3.2), necessita-se apenas de intervalos de confiança para as médias mensais, trimestrais e semestrais, que foram obtidos com sucesso a partir dos registros existentes.

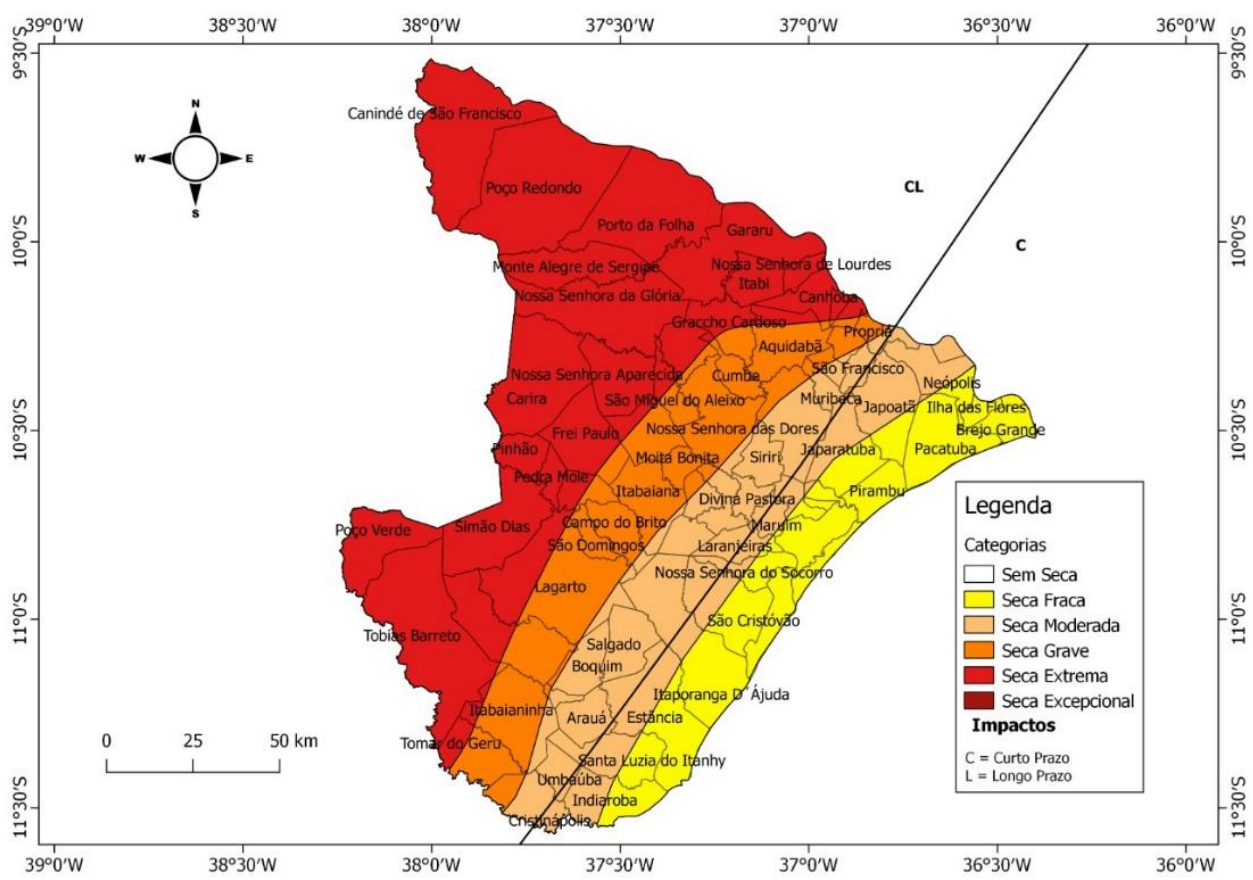

Figura 2 - Mapa do mês de novembro de 2018 para o estado de Sergipe.

Os valores médios mensais de NDVI por município foram obtidos por meio das imagens de satélite disponibilizadas pela Administração Nacional da Aeronáutica e Espaço dos Estados Unidos (NASA, National Aeronautics and Space Administration) (NASA, 2019). Foi utilizado o produto MOD13Q1 do sensor MODIS sob a forma das imagens do NDVI, com resolução de $250 \mathrm{~m}$ e composição de 16 dias. Foram adquiridas imagens desde a primeira quinzena de julho de 2018 até a última quinzena de outubro de 2019. O programa QGIS foi utilizado para o processamento dos dados.

\subsection{APLICAÇÃO DA ESTATÍSTICA E ANÁLISE DOS RESULTADOS}

Para verificar se há associação entre os dados climáticos disponíveis para os validadores e as categorias de seca consolidadas pelo MSB, foram utilizadas tabelas de contingência, o teste de independência do Qui-Quadrado e o coeficiente $V$ de Cramer, descritos a seguir.

\subsubsection{Tabelas de contingência}

Tabelas de contingência consistem em uma distribuição conjunta de frequência e são utilizadas quando os $n$ elementos de uma amostra podem ser categorizados por diferentes critérios, de modo a identificar se dois métodos de 
classificação estão estatisticamente associados (MONTGOMERY; RUNGER, 2014).

Para a construção das tabelas, considerando que o primeiro método tenha $r$ níveis e o segundo tenha $c, O_{i j}$ representa a frequência observada para o nível $i$ do primeiro método de classificação (linhas) e nível $j$ para o segundo (colunas). Assim, os dados, em geral, aparecem como na Tabela 1.

Tabela 1 - Tabela de contingência $r \times c$. Fonte: Montgomery e Runger (2014).

\begin{tabular}{ccccccc}
\hline & & \multicolumn{4}{c}{ Colunas } & \multirow{2}{*}{ Total } \\
\cline { 3 - 6 } & 1 & $O_{11}$ & $O_{12}$ & $\cdots$ & $O_{1 c}$ & $\sum_{j=1}^{c} O_{1 j}$ \\
\hline \multirow{2}{*}{ Linhas } & 2 & $O_{21}$ & $O_{22}$ & $\cdots$ & $O_{2 c}$ & $\sum_{j=1}^{c} O_{2 j}$ \\
& $r$ & $O_{r 1}$ & $O_{r 2}$ & $\cdots$ & $O_{r c}$ & $\sum_{j=1}^{c} O_{3 j}$ \\
\hline \multirow{2}{*}{ Total } & & $\sum_{i=1}^{r} O_{i}$ & $\sum_{i=1}^{r} O_{i}$ & $\cdots$ & $\sum_{i=1}^{r} O_{i}$ & $\sum_{i=1}^{r} \sum_{j=}^{c}$
\end{tabular}

De modo geral, as tabelas de contingência representam a distribuição conjunta de frequência das observações climáticas e categorias do Monitor para os meses de julho de 2018 a outubro de 2019, para todo o estado de Sergipe. A categorização das linhas corresponde aos níveis de seca do Monitor: sem seca, seca fraca, seca grave, seca moderada e seca extrema. Como não houve municípios em seca excepcional durante o período avaliado, essa categoria foi excluída. Para as colunas, foi considerada uma classificação própria para cada variável climática analisada.

É importante destacar que, como geralmente os totais marginais são diferentes, pode ser difícil interpretar os valores apenas com a distribuição das frequências (OGLIARI; ANDRADE, 2005). Desse modo, após a construção das tabelas de contingência, foram calculadas frequências relativas, em porcentagem, em relação ao total de cada coluna. Assim, pôde-se analisar a influência das variáveis climáticas na classificação do Monitor.

Para a precipitação (mensal, trimestral ou semestral), as colunas representaram as categorias "acima da média", "dentro da média" e "abaixo da média". De modo a conferir maior confiabilidade a essa análise, foram calculados intervalos de confiança $\left(\mathrm{IC}_{\mathrm{f}}\right.$ ) em torno da média para todos os meses e postos pluviométricos considerados.

Intervalos de confiança são determinados por dois números, calculados a partir de uma amostra, que contêm o valor de determinado parâmetro com nível de confiança $(1-\alpha) \%$, em que $\alpha$ é o nível de significância considerado (MARTINS; DOMINGUES, 2017).

Quando a variância da população é desconhecida, como é o caso das séries históricas de precipitação, 0 intervalo de confiança para média populacional é determinado por $\bar{X}-t_{\frac{\alpha}{2}} \frac{s}{\sqrt{n}} \leq \mu \leq \bar{X}+t_{\frac{\alpha}{2}} \frac{s}{\sqrt{n}}$ (MARTINS; DOMINGUES, 2017), em que $\bar{X}$ é a média amostral, $t_{\frac{\alpha}{2}}$ é a estatística $t$ de Student com $(n-1)$ graus de liberdade e nível de significância $\alpha, S$ é o desvio-padrão da amostra, $n$ 
é o número total de elementos da amostra e $\mu$ é a média populacional. Nesta pesquisa, foi considerado valor de $\alpha$ de $5 \%$, conforme usualmente adotado.

Assim, os valores observados de precipitação (mensal, trimestral e semestral) de julho de 2018 a outubro de 2019 para cada posto foram comparados ao $\mathrm{IC}_{f}$ da média mensal do município correspondente, classificados em "abaixo da média", "dentro da média" e "acima da média" e contabilizados na tabela de contingência.

Durante o processo de validação, a anomalia indica o quão distante da média se encontram as chuvas, tanto positiva quanto negativamente. Ou seja, a anomalia de precipitação mede os desvios de chuva em relação à média. Assim, a classificação adotada nas colunas das tabelas de contingência referentes à anomalia (mensal, trimestral ou semestral) foi a seguinte (sendo $S$ o desviopadrão da amostra):

- À esquerda do intervalo $(-S, S)$;

- Dentro do intervalo $[-S, S]$;

- À direita do intervalo $(-S, S)$.

Observe-se que, nesta etapa, os municípios sem postos pluviométricos em funcionamento não foram considerados. Assim, o número de observações na tabela de contingência foi de 400 ( 25 postos pluviométricos $\times 16$ meses).

Os valores do NDVI, índice de vegetação desenvolvido por Rouse et al. (1973), correspondem a uma medida quantitativa de condições da vegetação em grandes áreas e variam de 0 a 1 (quando relativos ao solo e à vegetação), indicando maior desenvolvimento e saúde vegetal quanto mais próximos de 1 . Nesse estudo, para as colunas da tabela de contingência, os valores médios de NDVI por município foram classificados em quatro intervalos iguais:

- $\quad$ De 0,00 a 0,25;

- De 0,25 a 0,50;

- De 0,50 a 0,75;

- De 0,75 a 1,00.

O número total de observações na tabela de categorias do MSB versus NDVI foi de 1200 (75 municípios $\times 16$ meses).

\subsubsection{Teste de independência do Qui-Quadrado e Coeficiente $V$ de Cramer}

O teste de independência do Qui-Quadrado é um teste de hipótese não paramétrico que objetiva determinar se há diferenças estatisticamente significativas entre as frequências observadas para dois grupos independentes. Essa técnica costuma ser utilizada para analisar a associação entre duas variáveis, podendo ser utilizada para dados nominais ou agrupados em intervalos (KRASKA-MILLER, 2014).

Como hipótese nula $\left(\mathrm{H}_{0}\right)$, tem-se que os métodos de classificação linha e coluna são independentes. Ao rejeitar essa hipótese, conclui-se que há interação entre os dois critérios de classificação (MONTGOMERY; RUNGER, 2014).

Seja $p_{i j}$ a probabilidade de um elemento aleatoriamente selecionado pertencer à célula $i j$, caso as classificações sejam independentes. Então $p_{i j}=$ $u_{i} v_{j}$, sendo $u_{i}$ a probabilidade de o elemento ser da linha $i$ e $v_{j}$ a probabilidade 
de o elemento ser da coluna $j$. Desse modo, se as classificações são independentes, os estimadores para $u_{i}$ e $v_{j}$ são:

$$
\begin{aligned}
& \hat{u}_{i}=\frac{1}{n} \sum_{j=1}^{c} O_{i j} \\
& \hat{v}_{j}=\frac{1}{n} \sum_{i=1}^{r} O_{i j}
\end{aligned}
$$

Havendo independência entre as classificações, a frequência esperada $\left(E_{i j}\right)$ para cada célula é dada por:

$$
E_{i j}=n \widehat{u}_{i} \widehat{v}_{j}=\frac{1}{n} \sum_{j=1}^{c} \boldsymbol{O}_{i j} \sum_{i=1}^{r} \boldsymbol{O}_{i j}
$$

em que $\sum_{j=1}^{c} O_{i j}$ e $\sum_{i=1}^{r} O_{i j}$ são totais marginais da tabela de contingência e $n$ é o número total de observações.

A estatística de teste $\chi_{0}^{2}$, dada pela Equação 4, tem uma distribuição aproximada Qui-Quadrado com $(r-1)(c-1)$ graus de liberdade se a hipótese nula for verdadeira (MARTINS; DOMINGUES, 2017).

$$
\chi_{0}^{2}=\sum_{i=1}^{r} \sum_{j=1}^{c} \frac{\left(o_{i j}-E_{i j}\right)^{2}}{E_{i j}}
$$

A hipótese de independência é rejeitada se $\chi_{0}^{2}$ for maior que $\chi_{\alpha,(r-1)(c-1)}^{2}$, valor tabelado, dado facilmente pela função inv.quiqua do programa Excel versão 2016, em que $\alpha$ é o nível de significância considerado. Nesse caso, conclui-se, $\operatorname{com}$ risco $\alpha$, que as variáveis são dependentes, ou estão associadas (MARTINS; DOMINGUES, 2017).

No caso de rejeição de $\mathrm{H}_{0}$, a probabilidade de as diferenças existentes entre as variáveis estudadas serem devidas ao acaso é pequena, ou seja, o valor-p, menor nível de significância que levaria à rejeição da hipótese nula a partir dos dados fornecidos, é muito pequeno (MONTGOMERY; RUNGER, 2014). Assim, se o valor-p, obtido por meio da função teste.quiqua do Excel 2016, é menor que $\alpha$, rejeita-se a $\mathrm{H}_{0}$.

Conforme Kraska-Miller (2014), no teste do Qui-Quadrado, um pequeno valor-p indica forte evidência de associação, mas provê pouca informação acerca da força da associação entre as variáveis. Por esse motivo, neste trabalho, foi calculado o coeficiente $V$ de Cramer, uma medida não-paramétrica de associação entre duas variáveis, que pode ser aplicada quando alguma das variáveis possui escala nominal e é dado pela Equação 5 (MARTINS; DOMINGUES, 2017):

$$
V=\sqrt{\frac{\chi_{0}^{2} / n}{\min (r-1),(c-1)}}
$$

em que $n$ é o número de observações, $r$ e $c$ representam o número de linhas e colunas, respectivamente, da tabela de contingência.

Esse coeficiente varia entre 0 e 1 , indicando o grau de associação ou dependência entre duas variáveis submetidas ao teste do Qui-Quadrado. Quanto mais próximo de 1 for o coeficiente $V$, maior a associação entre as variáveis, conforme indica o Quadro 1. 
Quadro 1 - Regra para interpretação do coeficiente $V$ de Cramer. Fonte: Adaptado de Kraska-Miller (2014).

\begin{tabular}{|c|c|}
\hline Coeficiente $V$ & Classificação \\
\hline 0 & Nenhuma associação \\
\hline$<0,30$ & $\begin{array}{c}\text { Associação muito fraca ou } \\
\text { insignificante }\end{array}$ \\
\hline 0,30 a 0,70 & Associação fraca a forte \\
\hline$>0,70$ & Forte associação \\
\hline 1 & Associação perfeita \\
\hline
\end{tabular}

Por meio do cálculo do $V$, associado ao resultado do teste do QuiQuadrado, foi possível analisar a significância da associação existente entre a representação de Sergipe pelo $M S B$ e as variáveis climáticas observadas no estado.

\section{RESULTADOS E DISCUSSÃO}

\subsection{TABELAS DE CONTINGÊNCIA}

Os dados de precipitação, anomalias e NDVI foram separados por município e mês e relacionados às categorias mensais do Monitor de Secas. Assim, foram construídas tabelas de contingência para cada variável.

Para fins de interpretação dos dados quanto à associação, foram calculadas, em porcentagem, as frequências relativas ao total de cada coluna. Assim, foi possível visualizar o percentual de cada categoria em relação ao total de casos de precipitação acima, dentro e abaixo da média. Já para as anomalias, foi calculado o percentual de cada célula em relação ao total à esquerda, dentro e à direita do intervalo de $-S$ a $S$; e, para o NDVI, o percentual de cada célula em cada uma das faixas de NDVI: 0,00 a 0,25, 0,25 a 0,50, 0,50 a 0,75 e 0,75 a 1,00 .

A Figura 3 apresenta os gráficos de frequência relativa de categorias do MSB versus precipitações. Nos três casos, observa-se que, de fato, chuvas abaixo da média têm maiores percentuais nas categorias mais severas da seca (grave e extrema). Contudo, percentuais consideráveis também se encontram nas categorias mais brandas e até mesmo sem seca. Para precipitações dentro da média histórica, houve municípios classificados nas diversas categorias. Já para chuvas acima da média, apesar do aumento percentual para categorias mais leves da seca, há valores ainda altos nas categorias moderada e grave.

Verifica-se ainda na Figura 3 que, nas três situações, ao passar de precipitações abaixo da média em direção a chuvas acima da média, há redução no percentual das categorias de seca extrema e grave, o que pode ser um indicativo de associação entre as variáveis. De modo semelhante, a categoria sem seca tem frequências maiores para chuvas dentro e acima da média. Ainda assim, apesar de haver algumas coincidências entre precipitações abaixo da média e categorias mais severas de seca, não há forte evidência de que a precipitação mensal tenha influenciado no traçado do Monitor. 
a) precipitações mensais

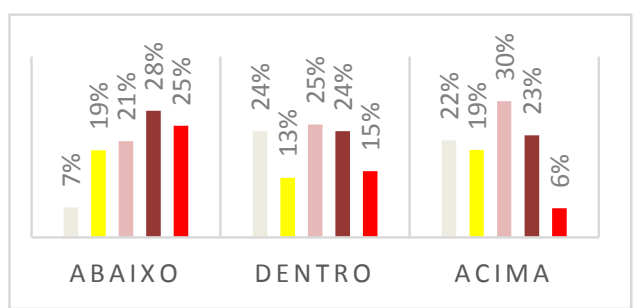

b) precipitações trimestrais

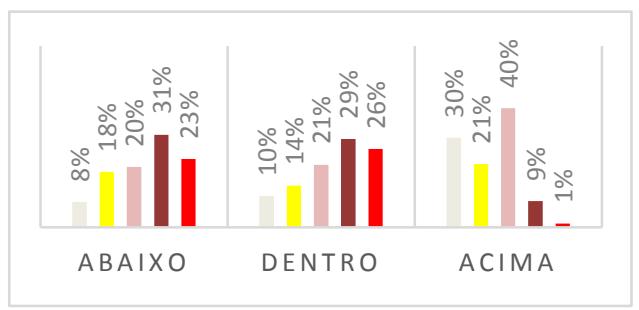

c) precipitações semestrais

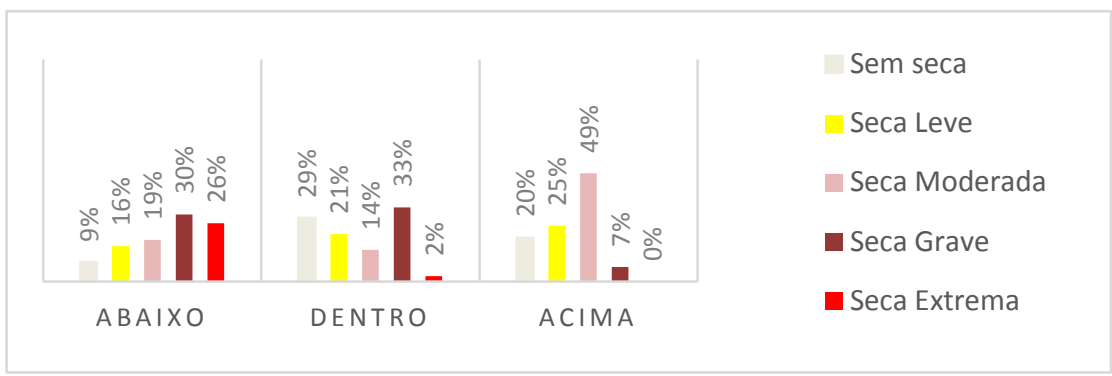

Figura 3 - Gráficos de frequências relativas - categorias do MSB vs precipitações a) mensais; b) trimestrais; c) semestrais nas categorias abaixo, dentro e acima da média.

Uma vez que o MSB apresenta a premissa de representação da seca relativa, que reflete a situação atual em relação ao histórico da região, esperase que chuvas acima da média contribuam para melhoria dos impactos da seca, ou seja, regiões com chuvas abundantes podem refletir uma classificação mais branda da seca, a depender do tipo de impacto resultante do balanço hídrico dos últimos meses. Por isso, períodos maiores de observação, como trimestrais e semestrais, mostram maior associação com as categorias do Monitor do que dados mensais. Exemplo disso são a redução significativa da seca extrema e o aumento da categoria sem seca ao passar de precipitações abaixo para precipitações acima da média nos itens b e c da Figura 3, referentes às precipitações trimestrais e semestrais, respectivamente.

Pela definição de seca física do Monitor, regiões com chuvas dentro do normal não deveriam ser consideradas em períodos de seca, diferentemente do que se vê nos gráficos apresentados. É possível que essas observações de seca durante épocas de chuvas normais sejam devidas ao fato de serem analisados, durante a confecção dos mapas, períodos maiores que um semestre. Além disso, outros parâmetros, como evapotranspiração, são também considerados pelo Monitor por meio dos índices de seca. Adicionalmente, deve-se destacar que são consideradas informações de apenas três postos pluviométricos para Sergipe, o que pode influenciar na correlação entre dados de chuva e categorias do Monitor.

A mesma análise percentual foi realizada para as anomalias de precipitação, cujos gráficos estão apresentados na Figura 4. 
a) anomalias mensais

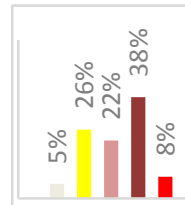

ESQUERDA INTERVALO b) anomalias trimestrais

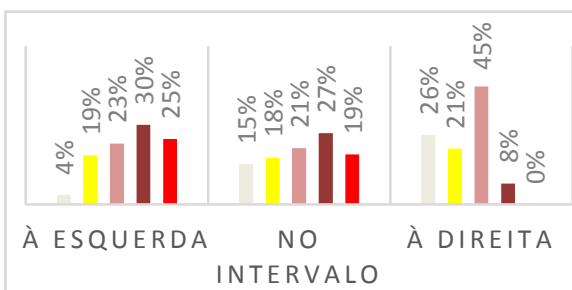

c) anomalias semestrais

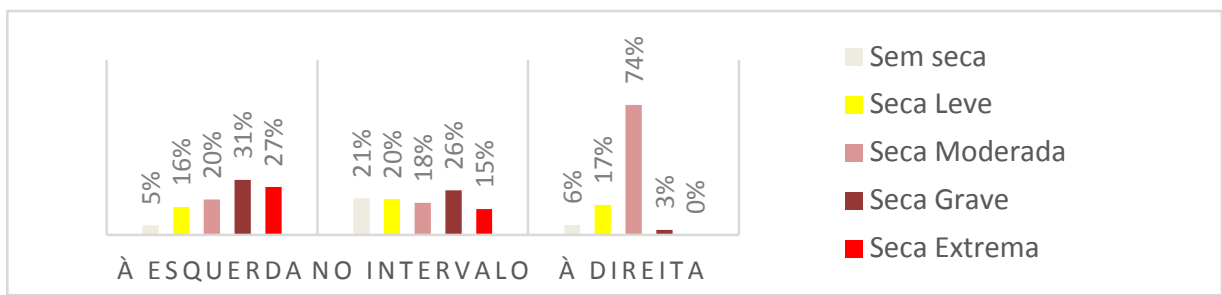

Figura 4 - Gráficos de frequências relativas - categorias do MSB vs anomalias a) mensais; b) trimestrais; c) semestrais.

Observa-se que, para anomalias à esquerda de $(-S, S)$, relacionadas a maiores desvios negativos em relação à média, a categoria predominante é a de seca grave, nos três casos. Contudo, valores consideráveis também ocorrem em outras categorias. De fato, havendo relação entre anomalia e categorias, espera-se que regiões com anomalias negativas de precipitação estejam passando por um período considerado seco.

Dentro do intervalo, todas as categorias apresentam percentuais semelhantes, com valores superiores nos níveis mais severos da seca, o que pode ocorrer devido à possibilidade de períodos maiores que um semestre refletirem desvio de chuvas muito abaixo da média, apesar de as anomalias do mês, trimestre ou semestre serem menores que o valor de um desvio padrão. Já à direita de $(-S, S)$, os percentuais mais altos concentram-se nos níveis mais brandos da seca, o que é esperado quando há associação entre as classificações. A tendência é que anomalias positivas de precipitação contribuam para redução de impactos da seca, o que, entretanto, não necessariamente será percebido imediatamente, visto que alterações na severidade da seca são lentas e graduais.

Ao sair da esquerda para direita de $(-S, S)$ nos gráficos, observa-se que, como esperado, o percentual de ocorrências sem seca aumenta, enquanto para os níveis de seca grave e extrema, os valores são reduzidos, o que é um indicativo de associação entre os mapas e dados de anomalia. Entretanto, devido à maioria das observações estar contida em $[-S, S]$, é difícil afirmar, apenas pela tabela de contingência, se realmente há associação entre os mapas e a anomalia mensal.

A Figura 5 apresenta o resultado da tabela de contingência para categorias do MSB versus NDVI. Nela, vê-se que para todos os casos de NDVI entre 0 e 0,25, a categoria correspondente foi a de seca extrema. A faixa seguinte, de 0,25 a 0,50, também possui maiores percentuais para categorias 
mais severas da seca. A partir do valor de 0,50, as duas últimas classes de NDVI contêm maiores percentuais para categorias mais brandas da seca. Assim, a distribuição de frequências dá um forte indício de associação entre as variáveis, uma vez que os valores de NDVI mais próximos de zero indicam regiões com solo exposto, ausência de folhas, pouca ou nenhuma cobertura vegetal, situação que, de fato, deve estar associada a níveis mais graves da seca. Já para valores de NDVI próximos de 1 , que representam regiões com melhores condições da vegetação, esperavam-se maiores percentuais nas categorias mais leves da seca.

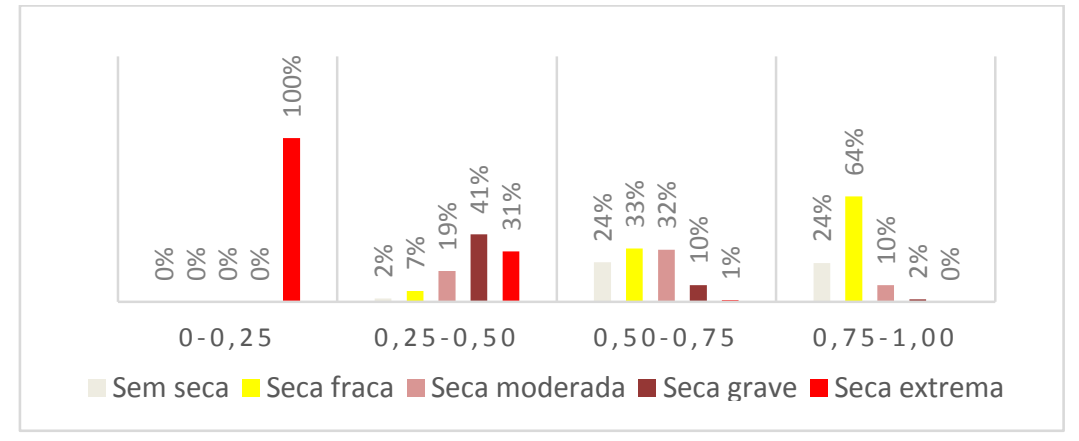

Figura 5 - Gráficos de frequências relativas - categorias do MSB vs anomalias a) mensais; b) trimestrais; c) semestrais.

De modo geral, percebe-se que apenas com a análise da tabela de contingência não é possível afirmar se há, realmente, associação entre as variáveis. Por esse motivo, foram aplicadas as ferramentas estatísticas do teste de independência do Qui-Quadrado e do coeficiente $V$ de Cramer para todos os casos em análise, cujos resultados são apresentados a seguir.

\subsection{TESTE DE INDEPENDÊNCIA DO QUI-QUADRADO E COEFICIENTE $V$ DE CRAMER}

Para os testes de independência realizados, teve-se como hipótese nula $\left(\mathrm{H}_{0}\right)$ que as categorias do Monitor são independentes da precipitação, anomalia ou NDVI observado. A hipótese alternativa $\left(\mathrm{H}_{1}\right)$, caso $\mathrm{H}_{0}$ fosse rejeitada, é que há associação entre precipitação/anomalia e categorias do MSB. Os resultados para todos os casos estudados estão apresentados na Tabela 2. 
Tabela 2 - Resultados do Teste do Qui-Quadrado e coeficiente V de Cramer.

\begin{tabular}{|c|c|c|c|c|c|c|c|}
\hline Parâmetro & $\begin{array}{c}\text { Precipitação } \\
\text { mensal }\end{array}$ & $\begin{array}{c}\text { Precipitação } \\
\text { trimestral }\end{array}$ & $\begin{array}{c}\text { Precipitação } \\
\text { semestral }\end{array}$ & $\begin{array}{c}\text { Anomalia } \\
\text { mensal }\end{array}$ & $\begin{array}{l}\text { Anomalia } \\
\text { trimestral }\end{array}$ & $\begin{array}{l}\text { Anomalia } \\
\text { semestral }\end{array}$ & NDVI \\
\hline$\alpha$ & $5 \%$ & $5 \%$ & $5 \%$ & $5 \%$ & $5 \%$ & $5 \%$ & $5 \%$ \\
\hline$n$ & 400 & 400 & 400 & 400 & 400 & 400 & 1200 \\
\hline$r$ & 5 & 5 & 5 & 5 & 5 & 5 & 5 \\
\hline$c$ & 3 & 3 & 3 & 3 & 3 & 3 & 4 \\
\hline$X_{a,(r-1)(c-1)^{2}}$ & 15,51 & 15,51 & 15,51 & 15,51 & 15,51 & 15,51 & 21,03 \\
\hline$X_{0}^{2}$ & 35,14 & 61,58 & 83,71 & 32,8 & 36,69 & 83,83 & 656,76 \\
\hline Resultado & $\begin{array}{c}\text { Rejeita-se } \\
\mathrm{H}_{0}\end{array}$ & $\begin{array}{c}\text { Rejeita-se } \\
\mathrm{H}_{0}\end{array}$ & $\begin{array}{c}\text { Rejeita-se } \\
\mathrm{H}_{0}\end{array}$ & $\begin{array}{c}\text { Rejeita-se } \\
\mathrm{H}_{0}\end{array}$ & $\begin{array}{c}\text { Rejeita-se } \\
\mathrm{H}_{0}\end{array}$ & $\begin{array}{c}\text { Rejeita-se } \\
\mathrm{H}_{0}\end{array}$ & $\begin{array}{l}\text { Rejeita } \\
\text {-se } \mathrm{H}_{0}\end{array}$ \\
\hline Valor-p & $2,5 \times 10^{-5}$ & $2,3 \times 10^{-10}$ & $8,7 \times 10^{-15}$ & $6,7 \times 10^{-5}$ & $1,3 \times 10^{-5}$ & $8,3 \times 10^{-15}$ & $\begin{array}{c}7,8 \times 1 \\
0^{-133}\end{array}$ \\
\hline V & 0,21 & 0,28 & 0,32 & 0,20 & 0,21 & 0,32 & 0,53 \\
\hline $\begin{array}{c}\text { Força da } \\
\text { associação }\end{array}$ & $\begin{array}{l}\text { Muito } \\
\text { fraca }\end{array}$ & $\begin{array}{l}\text { Muito } \\
\text { fraca }\end{array}$ & $\begin{array}{l}\text { Fraca } \\
\text { a forte }\end{array}$ & $\begin{array}{l}\text { Muito } \\
\text { fraca }\end{array}$ & $\begin{array}{l}\text { Muito } \\
\text { fraca }\end{array}$ & $\begin{array}{l}\text { Fraca } \\
\text { a forte }\end{array}$ & $\begin{array}{l}\text { Fraca } \\
\text { a forte }\end{array}$ \\
\hline
\end{tabular}

Em todas as análises, a hipótese nula foi rejeitada. Ou seja, significa que para o nível de significância adotado (5\%), pode-se afirmar que há associação entre as categorias de seca do MSB e os dados climáticos em questão.

$O$ valor-p associado ao teste de independência foi de $2,5 \times 10^{-5}, 2,3 \times 10^{-10}$ e $8,7 \times 10^{-15}$, para chuvas mensais, trimestrais e semestrais, respectivamente. Isso significa que há pequena chance de erro ao afirmar que os mapas e as precipitações não são independentes, ou seja, que possuem associação. Adicionalmente, observa-se que a confiabilidade na rejeição da hipótese nula aumentou à medida que o período observado passou de mensal para trimestral e semestral.

Ao calcular o coeficiente de Cramer, os valores encontrados foram 0,21 , 0,28 e 0,32 para associação entre categorias do MSB e precipitações mensais, trimestrais e semestrais, nessa ordem. Por estarem mais próximos de 0 do que de 1 , esses coeficientes demonstram que a força da associação analisada é fraca. Assim, pode-se afirmar que, apesar de haver relação entre os mapas do Monitor e as precipitações, a associação existente ainda é baixa.

Embora os resultados encontrados demonstrem fraca associação entre precipitação e representação da seca pelo MSB, em geral, com o aumento do período de chuva acumulada, houve maior correlação entre as classificações. Desse modo, pode-se afirmar que a associação dos mapas do MSB com dados de precipitação é mais forte para períodos maiores.

Pode-se atribuir a fraqueza dos resultados encontrados ao fato de que o Monitor de Secas se baseia em apenas três postos pluviométricos para análise das chuvas ocorridas no estado de Sergipe, o que leva a uma representação generalizada, que não considera a situação real existente na maioria das localidades. Além disso, deve-se destacar que o MSB também consulta dados de precipitação para períodos maiores que um semestre $(9,12,18$ e 24 meses) durante a elaboração dos mapas.

Quanto à análise da correlação entre as categorias de seca e as anomalias de precipitação, foram encontrados resultados semelhantes. $O$ teste do Qui-Quadrado levou a rejeição da hipótese de independência entre as 
variáveis, com valor-p de $6,7 \times 10^{-5}, 1,3 \times 10^{-5}$ e $8,3 \times 10^{-15}$ para anomalias de precipitação mensal, trimestral e semestral, respectivamente. Já o coeficiente $V$ assumiu valores de 0,20, 0,21 e 0,32, indicando fraca associação entre os mapas e as anomalias mensal, trimestral e semestral. Desse modo, confirma-se que, apesar de haver relação entre os mapas e anomalias de chuva, essa associação não é forte.

Assim como para as precipitações, os resultados para as anomalias foram melhores à medida que o período de tempo considerado foi maior. Esse é um indício de que os mapas do Monitor tendem a representar a seca principalmente por meio de observações de longo prazo.

Como a seca tem desenvolvimento gradual, de fato é esperado que mudanças de curto prazo, especialmente de precipitação, tenham menor influência sobre os impactos da seca e, consequentemente, sobre as categorias do MSB. Além disso, os autores do MSB buscam analisar não apenas os índices relacionados à chuva, como o SPI, mas também outros produtos que refletem os demais aspectos da seca, uma vez que análises isoladas podem levar a conclusões equivocadas sobre a situação existente. Ainda assim, precipitações são um importante parâmetro no monitoramento das secas e, para aumentar a qualidade das informações apresentadas no Monitor, a rede pluviométrica analisada deve ser a maior possível. Ressalta-se que, ao comparar os dados apresentados no presente estudo, deve-se considerar as ressalvas relativas às distinções metodológicas aqui descritas.

Entre as variáveis climáticas utilizadas na validação e estudadas nesta pesquisa, a que apresentou maior correlação com os mapas do Monitor foi o NDVI, como era esperado, devido ao fato de o NDVI refletir de modo mais amplo as condições de umidade do solo e o estágio fenológico das plantas relacionados à seca agrícola. O teste de independência, nesse caso, resultou na rejeição da hipótese nula com valor-p igual a $7,8 \times 10^{-133}$, consideravelmente inferior aos encontrados anteriormente. O coeficiente de Cramer obtido foi 0,52 , indicando uma relação mais forte entre os mapas e o NDVI.

O índice de vegetação reflete propriedades de crescimento de plantas, estando próximo de 0 quando há áreas secas, de solo exposto, e de 1 quando a vegetação está bem desenvolvida. Tais características estão relacionadas à seca agrícola, enquanto déficits de precipitação são relativos à seca meteorológica. Assim, pode-se afirmar que há maior associação dos mapas do Monitor aos impactos agrícolas da seca.

\section{CONCLUSÕES}

Este trabalhou buscou analisar a existência da associação existente entre os mapas do Monitor de Secas para Sergipe e os dados climáticos estaduais, visto que Sergipe é o menor estado do Brasil e os índices de seca utilizados durante a elaboração dos mapas mensais são calculados para apenas três postos no estado.

Baseado nas definições de seca física e relativa do Monitor, segundo as quais os mapas representam a seca a partir de características naturais e relacionadas ao histórico da região, era esperado que houvesse associação entre os dados climáticos e as categorias do MSB. De fato, em todos os casos 
estudados, a hipótese de independência foi rejeitada. Entretanto, a força da associação mostrou-se, em geral, fraca.

Por meio dos resultados encontrados, pode-se afirmar que a representação de Sergipe com apenas três postos pluviométricos tem sido insuficiente para refletir a seca o mais próximo possível da realidade do estado. Além disso, o fato de nenhum dos postos consultados estar no Semiárido pode trazer prejuízos à representação da região mais afetada pela seca.

Além do reduzido número de postos consultados, destaca-se que durante o processo de validação do Monitor em Sergipe dispõe-se apenas de dados climáticos mensais, o que dificulta a concordância com os mapas propostos. Dessa forma, sugere-se que, para melhorar o diálogo entre autores e validadores e, consequentemente, aumentar a veracidade das informações sobre Sergipe, além de serem acrescentadas informações de todos os postos pluviométricos ativos no estado aos dados de entrada do Monitor, os validadores devem utilizar-se também de informações climáticas de médio e longo prazos, bem como acrescentar dados e informações semelhantes àquelas utilizadas como produtos de apoio durante a autoria dos mapas do Monitor.

Por sua vez, a associação entre NDVI e as categorias do MSB foi consideravelmente superior à encontrada para dados de precipitação e suas anomalias. Assim, pode-se afirmar que o NDVI tem sido uma boa ferramenta para a etapa de validação. Por refletir características de seca agrícola, o NDVI é a variável que mais se aproxima dos mapas do Monitor, possivelmente porque o MSB considera a seca a partir de características físicas e condições climáticas locais de até 24 meses anteriores.

Diante dos resultados obtidos, pode-se afirmar que os mapas do MSB ainda necessitam de ajustes para representar as secas em Sergipe de maneira confiável, provendo informações que possam, de fato, ser utilizadas pelos usuários finais para fins de convivência com a seca. Do mesmo modo, a partir do conhecimento dos resultados encontrados neste estudo, os validadores estaduais estão em busca de novos dados e produtos de apoio semelhantes àqueles utilizados pelos autores dos mapas, a fim de dispor de informações cada vez mais confiáveis para a representação de secas em Sergipe.

O Monitor de Secas é uma ferramenta fundamental para o avanço da gestão de secas no Brasil, sendo o monitoramento o primeiro passo rumo à gestão de riscos no país. À medida que o instrumento se consolida como meio de suporte à decisão para políticas públicas, faz-se necessário que as informações apresentadas estejam bastante próximas à realidade. Sendo assim, melhorias no MSB fornecem subsídios para uma gestão de riscos mais eficiente.

\section{AGRADECIMENTOS}

Os autores agradecem à Coordenação de Aperfeiçoamento de Pessoal de Nível Superior (CAPES) pela bolsa de estudos concedida, à Superintendência Especial de Recursos Hídricos e Meio Ambiente de Sergipe (SERHMA) pela disponibilização dos dados, e aos membros do comitê técnico-científico do Monitor de Secas em Sergipe pela parceria nos estudos realizados. 


\section{REFERÊNCIAS BIBLIOGRÁFICAS}

ANA - AGÊNCIA NACIONAL DE ÁGUAS. O Monitor de Secas. 2019. Disponível em: <http://monitordesecas.ana.gov.br/>. Acesso em: 28 mar. 2020.

BANCO MUNDIAL. Monitor de Secas no Nordeste: em busca de um novo paradigma para a gestão de secas. 1. ed. Brasília: Grupo Banco Mundial, 2015, $124 \mathrm{p}$.

CUNHA, A. P. M. do A. et al. Desafios para a Consolidação de um Sistema de Alerta de Risco de Desastre Associado às Secas no Brasil. Sustentabilidade em Debate, [s. I.], v. 10, n.1, p. 60-76, 2019. DOI: 10.18472/SustDeb.v10n1.2019.19380

DEPEC - DEPARTAMENTO ESTADUAL DE PROTEÇÃO E DEFESA CIVIL. Plano estadual de proteção e defesa civil. Aracaju: DEPEC, 2021. Disponível em: <https://www.defesacivil.se.gov.br/wp-content/uploads/2021/01/PLANODEFESA-CIVIL-2021.pdf>. Acesso em: 04 abril 2021.

DIDAN, K. et al. MODIS Vegetation Index User's Guide. Arizona: University of Arizona, 2015.2 Disponível em: <https://vip.arizona.edu/documents/MODIS/MODIS_VI_UsersGuide_June_2015 _C6.pdf>. Acesso em: 9 dez. 2019.

HAO, Z.; SINGH, V. P.; XIA, Y. Seasonal drought prediction: advances, challenges, and future prospects. Reviews of Geophysics, [s. I.], v. 56, p. 108$141,2018$.

KIEM, A. S. et al. Natural hazards in Australia: droughts. Climatic Change, [s. I.], v. 139, n. 1, p. 37-54, 2016.

KRASKA-MILLER, M. Nonparametric statistics for social and behavioral sciences. [s.I.]: CRC Press, 2014.

MARTINS, E. S. et al. Monitor de Secas no Nordeste - o processo In: DE NYS, E.; ENGLE, N. L.; MAGALHÃES, A. R. (Org.). Secas no Brasil: política e gestão proativas. Brasília: Centro de Gestão e Estudos Estratégicos - CGEE; Banco Mundial, 2016b. 292 p.

MARTINS, G. de A.; DOMINGUES, O. Estatística geral e aplicada. 6. ed. São Paulo: Atlas, 2017.

MONTGOMERY, D. C.; RUNGER, G. C. Applied statistics and probability for engineers. 6. ed. Arizona: John Wiley \& Sons, 2014.

NASA - NATIONAL AERONAUTICS AND SPACE ADMINISTRATION. Earthdata Search. 2019. Disponível em: <https://search.earthdata.nasa.gov>. Acesso em: 29 nov. 2019.

OGLIARI, P. J.; ANDRADE, D. F. De. Estatística básica para ciências agrárias e biológicas: com noções de experimentação. Florianópolis: Universidade Federal de Santa Catarina, 2005.

ROCHA, A. F. Panorama da seca no estado de Sergipe: impactos e ações de enfrentamento. Parcerias Estratégicas, [s. I.], v. 22, n. 44, p. 181-200, 2017. 
ROUSE, J. W. et al. Monitoring vegetation systems in the Great Plains with ERTS. In: EARTH RESOURCES TECHNOLOGY SATELLITE-1 SYMPOSIUM, 3., 1973, Washington. Proceedings... Washington: NASA. Goddart Space Flight Center, 1973, p. 309-317. Disponível em: <https://ntrs.nasa.gov/search.jsp?R=19740022614>. Acesso em: 25 jun. 2019.

SANTOS, S. R. Q. dos; CUNHA, A. P. M. do A.; RIBEIRO-NETO, G. G. Avaliação de dados de precipitação para o monitoramento do padrão espaço-temporal da seca no Nordeste do Brasil. Revista Brasileira de Climatologia., [s. I.], v. 25, n. 15, p. 80-100, 2019. DOI: 10.5380/abclima.v25i0.

SEPLAG - SECRETARIA DE ESTADO DO PLANEJAMENTO, ORÇAMENTO E GESTÃO. Geografia de Sergipe. SEPLAG, 2014?. Disponível em: <http://observatorio.se.gov.br/geografia-e-cartografia/publicacoes-degeografia-e-cartografia/geografia-de-sergipe>. Acesso em: 15 maio. 2018.

SEPLAG - SECRETARIA DE ESTADO DO PLANEJAMENTO, ORÇAMENTO E GESTÃO. Sergipe em números: síntese 2018. Sergipe: SEPLAG, 2018. Disponível em: <http://observatorio.se.gov.br/estatistica/sergipe-emnumeros>. Acesso em: 15 maio. 2018.

SERGIPE. Secretaria de Estado da Inclusão, Assistência Social e do Trabalho. Departamento Estadual de Proteção e Defesa Civil. Situação de emergência: 2019. Aracaju: Defesa Civil, 2020. Disponível em: <https://www.defesacivil.se.gov.br/?page_id=228>. Acesso em: 10 jan. 2020.

SOARES, J. A. S.; BARBOSA, E. M. Políticas de acesso à água no Brasil: pensando a evolução das políticas de combate à seca no Semiárido. Revista Gestão e Sustentabilidade Ambiental, Florianópolis, v. 8, n. 4, p.443-467, 2019. DOI: $10.19177 /$ rgsa.v8e42019443-467.

WMO - WORLD METEOROLOGICAL ORGANIZATION. Drought monitoring an early warning: concepts, progress and future challenges. WMO no 1006. 2006. 24 p 